\title{
XXV. On capillary phenomena
}

\author{
John T. Riley B.Sc. A.R.C.Sc.I.
}

To cite this article: John T. Riley B.Sc. A.R.C.Sc.I. (1883) XXV. On capillary phenomena, Philosophical Magazine Series 5, 15:93, 191-198, DOI: 10.1080/14786448308628454

To link to this article: http://dx.doi.org/10.1080/14786448308628454

$$
\text { 册 Published online: } 28 \text { Apr } 2009 .
$$

Submit your article to this journal

LII Article views: 3

Q View related articles ¿ 
foundation of his exposition of the subject, he would have removed what I take to be the only serious blemish in his excellent book.

In conclusion, I would point out that the wider scope here sought to be given to the fundamental theorems of Statics can be justified on other grounds. It is now generally held that the ultimate particles of bodies apparently at rest are really in a state of more or less violent agitation. If we regard Statics as the theory of equilibrium, and if by equilibrium we mean relative rest, there is a manifest awkwardness in applying the principles established on this basis to bodies so constituted. On the other hand, in the form advocated in this paper, the fundamental theorems are directly applicable to such cases, without any modification whatever.

Adelaide, November 25, 1882.

XXV. On Capillary Phenomena. By JoHn T. Riley, B.Sc. (London), A.R.C.Sc.I., Demonstrator in the Physical Laboratories of the Mason College*.

[Plate IV.]

A LTHOUGH the mathematical theories of capillary action and others agree in one point, which may be tested by experiment, they differ in the fundamental hypotheses. In all the theories the equation of the capillary surface is of the same form, involving a certain constant which can be determined by experiment only. They, however, differ in the manner in which this constant is made to depend on the molecular forces and the law of density of the fluid near the surface.

Laplace $\dagger$ supposes the density of the fluid to be uniform: he commences by considering an infinitely slender canal of the fluid perpendicular externally to the surface of a sphere, and calculates the total action of the sphere on the canal. The law of attraction is such a function of the distance that this function becomes insensible when the distance becomes sensible. He finds that the resultant attraction may be represented in the form $\mathrm{K}-\frac{\mathrm{H}}{b}$, where $\mathrm{K}$ and $\mathrm{H}$ are both independent of $b$, the radius of the sphere. $\mathrm{He}$ observes that $K$ is much larger than $\frac{H}{b}$, and that $K$ repre-

* Communicated by the Author.

† Mécanique Céleste, Supplément au Livrex. 
Phil Mag S 5 Vol 15 F. Ir
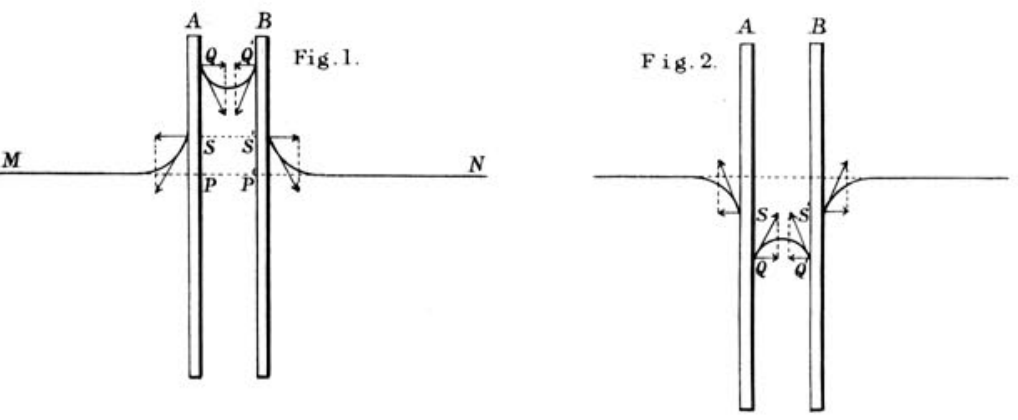

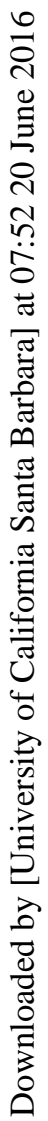
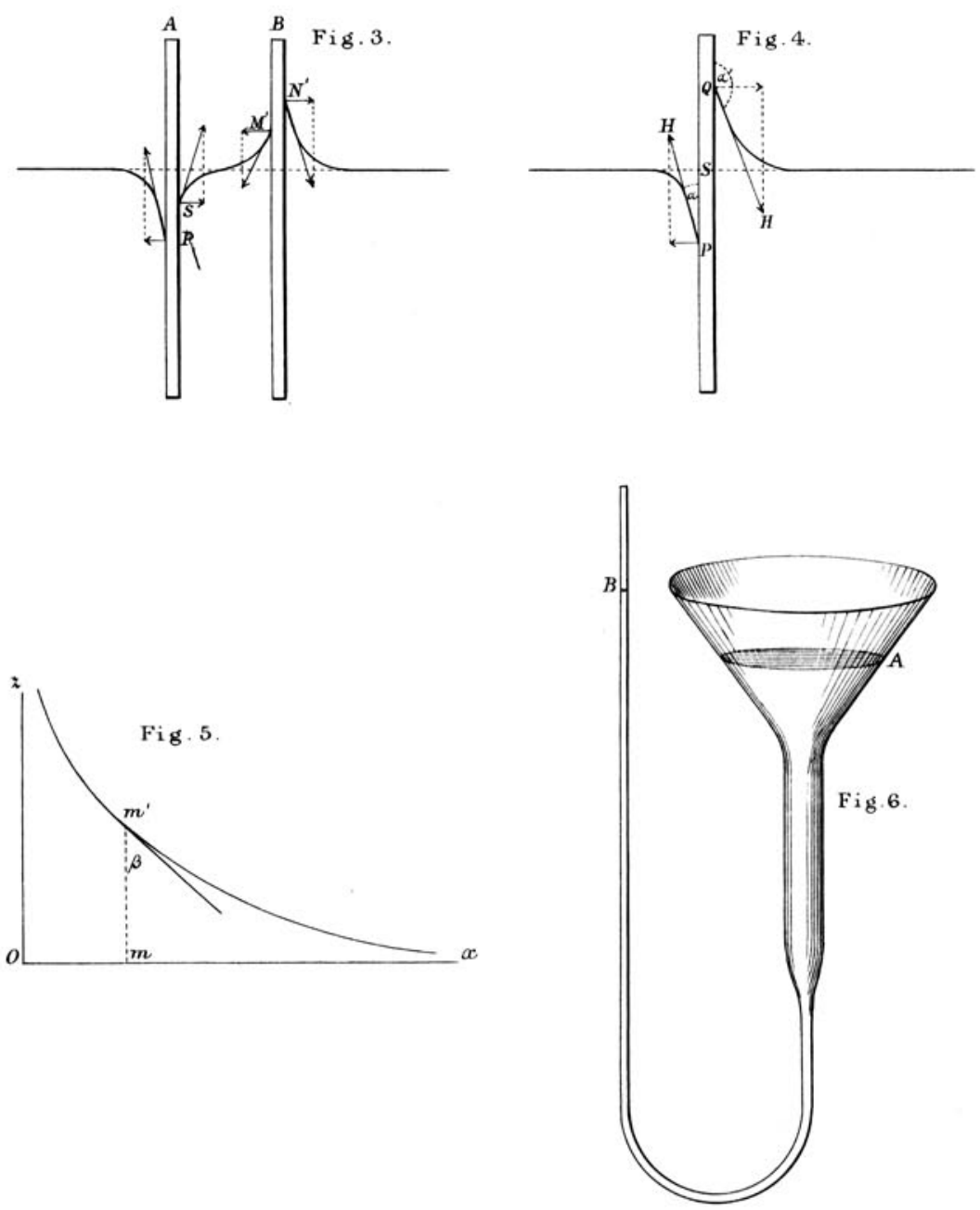
sents the attraction of a plane surface, since $\frac{H}{b}$ vanishes when $b$ becomes infinite. The expression $\mathrm{K}-\frac{\mathrm{H}}{b}$ also represents the attraction exerted on the canal by a segment cut from the sphere by a plane to which the canal is perpendicular, since all the sphere behind the plane is at too great a distance to have any effect. In a similar way, $\mathrm{K}+\frac{\mathrm{H}}{b}$ represents the action of a sphere upon an infinitely slender canal internally perpendicular to its surface.

$\mathrm{He}$ then applies this result to determine the action of any curved surface upon an internal column of fluid enclosed in an infinitely slender canal perpendicular to any point of this surface. If $R$ and $R^{\prime}$ be the principal radii of curvature at this point, he obtains for the attraction the expression

$$
\mathrm{K}+\frac{1}{2} \mathrm{H}\left(\frac{1}{\mathrm{R}}+\frac{1}{\mathrm{R}^{\prime}}\right) \text {. }
$$

Gauss * uses the principle of virtual velocities. He forms an expression for the sum of the potentials arising from the mutual action between pairs of particles. This expression consists of three parts, corresponding to the action of gravity, the mutual action between the particles of the fluid, and the action between the particles of the fluid and the particles of the solid or fluid in contact with it. The condition that the system may be in equilibrium is that this expression shall have a minimum value.

Poisson $\dagger$ maintains that the density of the fluid is not uniform, but that there is a rapid variation of density near the surface. $\mathrm{He}$ obtains an equation of the capillary surface similar to that of Laplace, but asserts that $K$ is very small instead of very grent.

Many physicists, unwilling to grant the possibility of a negative pressure in a liquid, and confronted with the fact that capillary elevations and depressions occur in vacuo just as in the atmosphere, adopt the result of Laplace, and consider that a plane surface exerts a considerable pressure on the interior liquid. Thus in Everett's edition of Deschanel (6th ed. p. 133) we find the following statement:- "We cannot conceive of negative pressure existing in the interior of a liquid, and we are driven to conclude that the elevation is owing to the excess of pressure caused by the plane

* Principia Generalia Theoria Figura Fluidorum in Statu Aquilibrii.

† Nouvelle Theorie de l'Action capillaive: Paris, 1831. 
surface in the containing vessel above the pressure caused by the concave surface in the capillary tube."

This molecular pressure, due to the plane surface, is a result only of the mathematical methods that have been employed to give an explanation of capillary phenomena. We cannot demonstrate experimentally the existence of this pressure, since, if introduced into the calculations at the beginning, it is afterwards eliminated and does not appear in the results.

In order to determine whether this molecular pressure, due to a plane surface, has any physical existence, I have made the following experiment, which I think conclasively proves that it has not, or, if it has, that it must be very small. I took a small funnel and drew out the stem so as to form a fine capillary tube which I bent twice at right angles, so as to bring the capillary limb parallel with the stem (fig. 6). The funnel was clamped to a stand, and water poured in to the level $A$; in the capillary limb it rose to $B$, the height of which I read with a cathetometer. Adopting Laplace's formula, and neglecting the atmospheric pressure, as its effect cancels out, the pressure in the liquid immediately under the plane surface at the level $\mathrm{A}$ is $\mathrm{K}$, and that immediately under the curved surface at $B$ is $K-\frac{H}{2}\left(\frac{1}{R}+\frac{1}{R^{\prime}}\right)$, since $R$ and $R^{\prime}$ are
both negative.

In this case $K$ cancels out; but if, without altering the level at $A$, we modify the surface tension, then $K$, which is, according to Laplace, a function of $\mathrm{H}$, is altered, and takes a value $K^{\prime}$. The excess of the pressure immediately below $\mathbf{A}$ over that immediately below $B$ is now $\mathrm{K}^{\prime}-\mathrm{K}+\frac{\mathrm{H}}{2}\left(\frac{1}{\mathrm{R}}+\frac{1}{\mathrm{R}^{\prime}}\right)$, instead of $\frac{\mathrm{H}}{2}\left(\frac{1}{\mathrm{R}}+\frac{1}{\mathrm{R}^{\prime}}\right)$ as before, and the capillary surface at $B$ ought to move up or down according as $K^{\prime}-K$ has a positive or negative value.

To test this, I held a glass rod, with a drop of ether adhering, close to the surface of water in the funnel: the surface tension was immediately diminished; but an observation with the cathetometer showed that no fall had occurred at B. Even when I dropped the ether on the surface I could detect no movement; but immediately I held the drop over the capillary tube, the surface at B began to sink, and finally fell several millimetres.

It is evident that, although $\mathrm{H}$ is very much diminished by the solution of the ether vapour in the capillary surface, the difference between $K$ and $K^{\prime}$ is not appreciable, so that both must be exceedingly sinall or zero. 
We may suppose that, while the drop of ether is held over the plane surface, the constitution of the liquid near the surface is continually changing, from the passage of the ether molecules into the upper layers.

The term $K^{\prime}$ is meant to include the whole of whatever plane-surface actions may exist, tending to produce an internal pressure. It is evident that $K^{\prime}$ ought to be considerably less than $\mathrm{K}$, since $\mathrm{H}^{\prime}$ is very much less than $\mathrm{H}$ under the same conditions of surface.

The equation of the capillary surface is then practically reduced to

$$
p=\frac{\mathrm{H}}{2}\left(\frac{1}{\mathbf{R}}+\frac{1}{\mathbf{R}^{\prime}}\right)
$$

where $p$ is the hydrostatic pressure immediately below the capillary surface.

All that we know experimentally concerning the forces which produce capillary phenomena may be expressed in two fundamental propositions, from which all explanations of particular phenomena may be deduced. It has been abundantly shown, by the experiments of Plateau, Dupré, Van der Mensbrugghe, Terquem, and many others, that every liquid behaves as if its external layer were in a state of tension. This tension is uniform in all directions in the free surface; but its magnitude is modified by contact with the surface of a solid or another liquid. The existence of this surface-tension is the basis of the physical explanation of capillary phenomena. The only assumption it seems necessary to make-namely, that the variations of pressure in the parts of the liquid elevated above the level of the free surface obey the ordinary laws of hydrostatics-seems so evidently natural that it cannot be doubted.

Commencing with these data, it is easily proved, as if for a stretched membrane, that the intensity of the pressure supported by a meniscus is given at any point in the meniscus by the expression $p= \pm H\left(\frac{1}{\mathrm{R}}+\frac{1}{\mathrm{R}^{\prime}}\right)$, where $\mathrm{R}$ and $\mathrm{R}^{\prime}$ are the principal radii of curvature at that point, and $H$ is the uniform tension across a linear centimetre in the surface.

The constancy of the angle of contact of a liquid with a given solid also follows easily from the assumption of a surface-tension; and thus armed we are able to explain any capillary phenomena.

In the January number of this Magazine, Prof. John Le Conte attempts to refer to two fundamental principles the explanation of several cases of the apparent attractions and repulsions of small floating bodies. He refers these phe- 
nomena exclusively to the elastic reactions of the tense surface-film, whose form is modified by the proximity of the partly immersed solid bodies, and purposely leaves out of consideration the modifications of hydrostatic pressure. Of course it is evident that these phenomena are primarily produced by the elastic reactions of the capillary surfaces in the neighbourhood of the solid bodies; but to consider the motions as produced by a "superior tension" in one or more of the films, owing to a smaller radius of curvature of the film, is evidently an error of expression.

The tension is constant in every direction throughout the capillary surfaces; and it is clear that, since for each plate the angle of contact is the same on both sides, the horizontal components of the tensions will balance each other. The movements of the bodies are secondary results of the surface-tension, being produced by the modified hydrostatic pressures on their surfaces.

In the case of the compound plate of steel and glass, Prof. Le Conte's treatment has evidently led him into an error ; for he says "It is obvious that the tensile reaction can only tend to press the plates together: it cannot produce a motion of translation." On the contrary, as I shall show, the effect of the tensile forces is to tend to pull the plates asunder; but these opposite forces being equal in amount, no movement of the plate ensues. In order to gain a clear physical conception of the reasons for these apparent attractions and repulsions, it is necessary to consider all the forces at work; and that I propose to do in the following explanations:-

Case I. (fig. 1). Let A and B be two flat plates partially immersed in a liquid which wets both. If the plates be brought sufficiently near each other, the capillary surface $\mathrm{QQ}^{\prime}$ will rise above the ordinary level of the liquid outside the plates. Let MN be drawn in the horizontal surface: if we neglect the atmospheric pressure, the hydrostatic pressure at this level will be zero. Passing upwards the pressure becomes negative, decreasing according to the ordinary law. There is thus a reaction of the nature of a tension between the liquid above MN and the solid surfaces in contact with it. From $\mathrm{P}$ to $\mathrm{S}$ the hydrostatic tension between the surfaces on the left of $\mathrm{A}$ will be balanced by the tension between the surfaces on the right; but from $S$ to $Q$ the tension towards the right is unbalanced by any tension towards the left. Since the angle of contact of the liquid with the solid is the same on both sides of $A$, the horizontal components of the surface-tensions are equal and in opposite 
directions, so that they can produce no horizontal motion. The resultant force on $A$ is thas the tension between the liquid surface $S Q$ and the solid ; and this force tends to move A towards B. Similarly, the resultant force on B is the tension between the liquid surface $S^{\prime} Q^{\prime}$ and the solid; and this force moves $\mathrm{B}$ towards $\mathrm{A}$. The two bodies are thus drawn together.

Case II. (fig. 2). In this case the liquid surface is depressed in the neighbourhood of both bodies, and, as before, the horizontal components of the surface-tension where the meniscuses touch the solids cancel out. It is at once evident that the hydrostatic pressures from $S$ to $Q$ and from $S^{\prime}$ to $Q^{\prime}$ on the external faces of $A$ and $B$ are unbalanced; and they therefore press the bodies together.

Case III. (fig. 3). Here we find a depression of the surface near one plate, and an elevation near the other. The horizontal components of the tensions on both sides of $A$ and $B$ will respectively destroy each other: the resultant hydrostatic pressure from $P$ to $S$ forces $A$ towards the left, and the resultant tension from $M^{\prime}$ to $N^{\prime}$ pulls $B$ to the right, and the plates tend to separate.

Case IV. (fig. 4). We have now to consider the equilibrium of a single floating plate, with its surfaces so prepared that they produce different degrees of capillary elevation. It is very easy to see that, in an indefinite surface of the liquid, no movement can take place. For the potential energy of the surface cannot be diminished by any movement of the plate; and therefore we conclude that the plate must be in equilibrium, for otherwise its kinetic energy when moving would have been produced without any diminution of potential energy. But it is important to show that we arrive at the same conclusion from an examination of the effects of the capillary forces brought into play. The case does not offer any ground for objection to Laplace's theory, as Dr. Thomas Young insisted it did, nor to any mathematical theory from which the fundamental equation given previously can be deduced.

Let the surfaces of the compound plate (fig. 6) be such that we have on one side a capillary depression and on the other a capillary elevation. For the equilibrium of the plate we must have the horizontal component of tension $\mathrm{H}$ at $\mathrm{Q}+$ hydrostatic tension from $S$ to $Q=$ horizontal component of tension $\mathrm{H}$ at $\mathrm{P}+$ hydrostatic pressure from $\mathrm{S}$ to $\mathrm{P}$. In order to determine the hydrostatic tension and pressure, we first find the pressure at any point in the curved surface. As $\mathrm{R}^{\prime}$ is infinitely great, the general equation reduces to $p=g \rho z$ 
$=\frac{\mathrm{H}}{\mathbf{R}}$, where $z$ is the height of the point considered ( $m^{\prime}$, fig. 5 ) from the axis of $x$ taken in the level surface. From the differential equation of the curvature at the point $m^{\prime}$ we get

and, integrating,

$$
\frac{\frac{d^{2} z}{d x^{2}}}{\left\{1+\left(\frac{d z}{d x}\right)^{2}\right\}^{\frac{3}{2}}}=\frac{1}{\mathrm{R}}=\frac{g^{\rho z}}{\tilde{\mathrm{H}}}
$$

$$
-\frac{\mathrm{H}}{\sqrt{1+\left(\frac{d z}{d x}\right)^{2}}}=\frac{g \rho z^{2}}{2}+\text { const. }
$$

When $z=0, \frac{d z}{d x}=0$, and const. $=-\mathrm{H}$, so that the equation becomes

$$
\mathrm{H}\left\{1-\frac{1}{\sqrt{1+\left(\frac{d z}{d x}\right)^{2}}}\right\}=\frac{g \rho z^{2}}{2} \text {. }
$$

Introducing the angle $\beta$ made by the tangent to the surface with the ordinate $z$, we get finally

$$
z= \pm \sqrt{\frac{2 \mathrm{H}}{g \rho}(1-\sin \beta)} . \quad . \cdot
$$

Now let the surface at $\mathrm{P}$ make an angle $\alpha$ with the surface of the plate, at $Q$ an angle $\alpha^{\prime}$. The horizontal components of the tensions will be $\mathrm{H} \sin a$ and $\mathrm{H} \sin \alpha^{\prime}$ respectively. The hydrostatic tension on the plate from $\mathrm{S}$ to $\mathrm{Q}$ will be

$$
\int_{0}^{\mathrm{SQ}} g \rho z d z=\frac{g \rho(\mathrm{SQ})^{2}}{2}=\mathrm{H}\left(1-\sin \alpha^{\prime}\right)
$$

from formula (1).

Similarly the hydrostatic pressure from $\mathrm{S}$ to $\mathrm{P}$ is $\mathrm{H}(1-\sin a)$; supplying these valuess in the equation for equilibrium we have the identity

$$
\mathrm{H}=\mathrm{H} \text {. }
$$

We thus see that the resultant forces acting on the plate are two equal and opposite tensions which will tend to pull the plates asunder. In every case the identity shows that the magnitude of these tensions is just the same as if the surfaces came up perpendicular to the plates without elevation or depression.

In conclusion I would point out that the mathematical theories of Laplace, Gauss, and Poisson do no more than 
account for a surface-tension. The investigations which Laplace gave of the rise of liquids in capillary tubes and between plates, and of the apparent attractions and repulsions of small floating bodies, cannot be considered as giving definite support to his theory, since the existence of the phenomena may be deduced from any mathematical theory which explains surface-tension.

All that Laplace did was to show that, with certain assumptions respecting the density of the liquid near the surface and the law of molecular attractions, he could prove that capillary phenomena would occur.

With other assumptions, Poisson also showed they would occur; but whether his assumptions or those of Laplace have a physical reality is an altogether different matter.

The Mrson College, February 5th.

XXVI. On the Horizontal Motion of Floating Bodies under the Action of Capillary Forces. By A. M. Worthington.

[Plate V.]

To the Editors of the Philosophical Magazine and Journal. Gentlemen,

WITH respect to the phenomena of capillary attraction and repulsion of floating bodies, which are the subject of Mr. J. LeConte's paper in the Phil. Mag. of January last, I would remark that in most writings on capillarity in which the elevation or depression of liquids in contact with solid plates is discussed, attention is confined to the vertical component of the surface-tension at its contact with the solid, and to its relation with the weight of liquid elevated or depressed. The horizontal component is generally neglected as not affecting the results; and the very interesting relation which exists between its variation and the resulting hydrostatic pressure or tension is overlooked. The consequence of this is that the explanations which are afterwards given of the horizontal motions of floating bodies are expressed in terms which apply only to each special case as it is dealt with, and do not reveal the important underlying relationship to which I refer.

This relationship is indeed made use of by Quincke in treating of flat drops and bubbles; and he deduces it from the differential equation to the surface by a rather long process. It does not seem to have occurred to any one that the same relationship underlies the attraction and repulsion of plates, or that it can be very easily deduced from the well-established 